\title{
Une nouvelle lettre de contact pour les hyménoptéristes apidologues francophones
}

\author{
Par Nicolas VEREECKEN *, Denis MICHEZ ** et Gilles MAHE ***
}

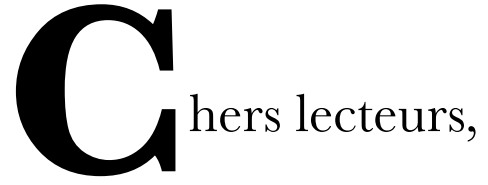

Vous tenez entre vos mains (ou vous lisez sur votre écran si vous consultez le PDF) le premier numéro d'OSMIA, fruit d'une collaboration entre passionnés d'abeilles sauvages en Europe francophone. Cette lettre de contact des apidologues a pour objectif principal la communication des observations réalisées par les membres du groupe Apoidea Gallica et la diffusion d'informations relatives à l'écologie, la systématique et l'évolution de ces insectes dont les adaptations à leur environnement offrent d'innombrables perspectives d'observations et de recherches.

L'étude des abeilles sauvages en Europe francophone n'est pas née d'hier: elle a été initiée dès la fin du XVIII ${ }^{\text {ème }}$ siècle par plusieurs entomologistes tels que Brullé, Latreille ou encore Pérez gravitant autour du Museum National d'Histoire Naturelle (MNHN) de Paris. Plus récemment, en France, des études de pollinisation des cultures agricoles par les abeilles sauvages furent menées par l'équipe de Jean-Noël Tasei et André Pouvreau, tous deux installés à l'Institut National pour la Recherche Agronomique (INRA) de Lusignan. Des recherches similaires de pollinisation appliquée sont aujourd'hui réalisées par l'équipe du Laboratoire de Pollinisation Entomophile de l'INRA d'Avignon sous la direction de Bernard Vaissière. En Belgique, les études portant sur l'apidofaune sauvage se sont développées bien plus tard, dans le courant des années 1970 autour de Jean Leclercq à la Faculté des Sciences Agronomiques de Gembloux et de Jean-Jules Pasteels à l'Université Libre de Bruxelles. Les successeurs du Pr Leclerq ont perpétué cette tradition à Gembloux (Sébastien Patiny, Alain Pauly, Annie Remacle) tout en développant un second pôle d'apidologie à l'Université de Mons-Hainaut, sous l’impulsion de Pierre Rasmont (Yvan Barbier, Michaël Terzo).

\footnotetext{
* Service d'Eco-Ethologie Evolutive, Université Libre de Bruxelles CP 160/12, Av. F.D. Roosevelt 50, B-1050 Bruxelles, Belgique.

E-mail: nicolas.vereecken@ulb.ac.be

** Laboratoire de Zoologie, Université de Mons-Hainaut, Av. Maistriau 19, B-7000 Mons, Belgique.

E-mail: denis.michez@umh.ac.be

*** Gilles Mahé, Rue de la matte 18, F-44600 Saint-Nazaire, France.

E-mail: gilles.mahe@wanadoo.fr
}
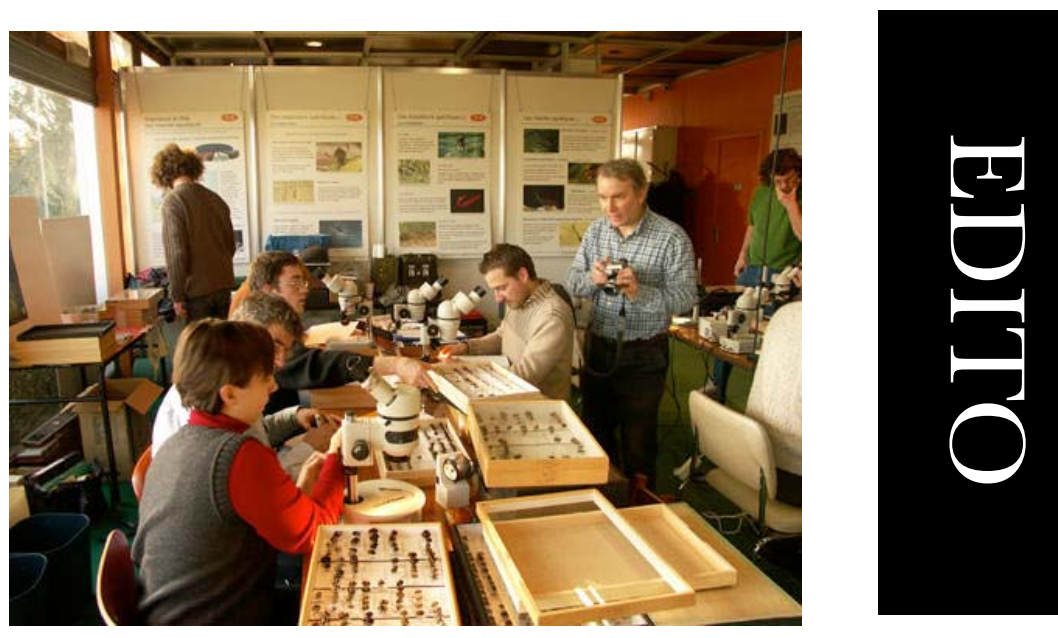

Figure 1. Rencontre Apoidea-Gallica à Guyancourt (France), 21.I.2006 (Photo H. Mouret)

Au fil des décennies, ces équipes de recherche et d'autres en Europe et dans le monde ont contribué à accumuler des connaissances sur la biologie, l'écologie, la systématique et l'évolution des abeilles sauvages en Europe. L'ensemble de ces informations ont éveillé l'intérêt pour l'apidofaune sauvage auprès du grand public, notamment via la publication d'ouvrages remarquables comme Die Wildbienen Baden-Württembergs de Westrich (1990) (voir aussi les références-clés dans la rubrique "Forum"), qui ont permis à de nombreux naturalistes de porter un autre regard sur les abeilles sauvages de nos régions. En outre, des études récentes et des recherches en cours confirment le rôle-clé des abeilles sauvages l'équilibre des écosystèmes, tant en milieu naturel qu'agricole. Malheureusement, il demeure qu'une proportion importante de l'apidofaune de nos régions est méconnue et menacée, notamment par la pression anthropique qui pèse sur l'intégrité de leurs sites de nidification et sur l'intégrité des populations des plantes à fleurs dont les abeilles sauvages dépendent exclusivement pour leur développement. Une étude récente portant sur l'évolution de la diversité floristique et entomologique au cours des 25 dernières années en Angleterre et aux Pays-Bas a mis en évidence un déclin significatif des populations d'abeilles sauvages, conséquence immédiate de la disparition des populations de plantes à fleurs auxquelles elles sont liées (Biesmeijer et al. 2006). 


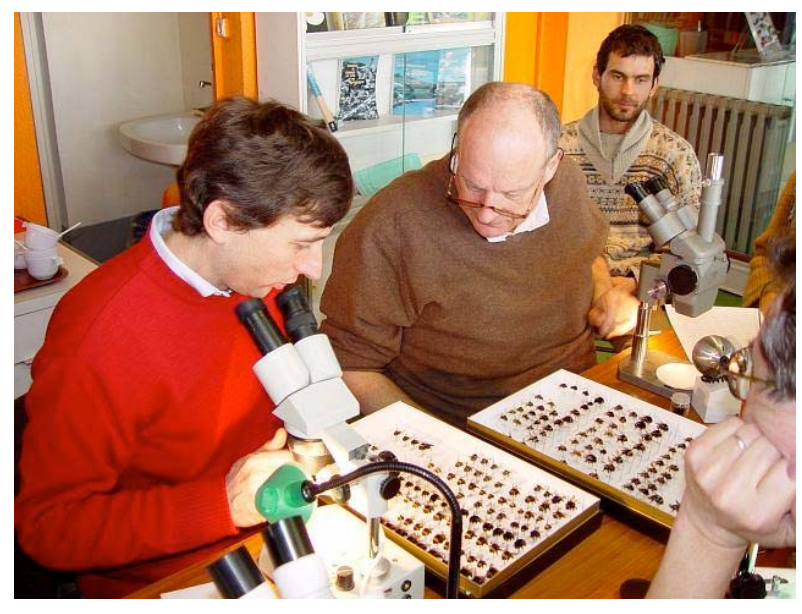

Figure 2. Rencontre Apoidea-Gallica à Guyancourt (France), 21.I.2006 (Photo H. Mouret)

C'est pour mettre en relation tous ceux qui s'intéressent aux abeilles sauvages, dans le but d'approfondir les connaissances relatives aux différentes espèces (biogéographie, systématique, écologie) qu'un groupe de discussion s'est créé en 2002 à l'initiative de naturalistes du massif armoricain (Peter Stallenger, Gilles Mahé, Gwenaël Lebras). Ce groupe, initialement baptisé Apoidea-Armoricana, a connu une croissance constante depuis sa création et comporte aujourd'hui des membres de France, de Belgique et d'Angleterre. Fort d'une centaine de membres et rebaptisé ApoideaGallica depuis 2006, le groupe se réunit chaque année dans le courant du mois de janvier, l'occasion pour les membres de rencontrer les nouveaux venus et d'échanger des données et de la documentation sur les abeilles sauvages. La participation d'experts internationaux en systématique et en écologie des abeilles sauvages contribue significativement à l'intérêt de ce type de réunion.

En 2006, certains membres du groupe ont émis l'idée de concevoir une lettre de contact originale qui serait faite par et pour les membres du groupe. Voici donc le premier numéro d'OSMIA, la lettre de contact des hyménoptéristes apidologues francophones.
Ce bulletin est né de l'initiative conjointe de plusieurs membres désireux de créer un homologue francophone à d'autres bulletins européens tels que le BZZZ hollandais, le bembix allemand ou encore la BWARS Newsletter anglaise. La vocation principale d'OSMIA est de publier des observations originales, mais aussi des commentaires sur des recherches en cours (via la rubrique "Forum") ou toute autre information susceptible d'intéresser la communauté.

Dans un premier temps, OSMLA sera publié à raison d'un numéro par an. Cette dynamique de publication sera progressivement adaptée en fonction du nombre d'articles proposés au comité de rédaction (voir rubrique "Aux Auteurs" du présent document). La diffusion de cette lettre d'information sera gratuite, en couleurs et se fera exclusivement par voie électronique (fichiers .pdf). Nous encourageons vivement les lecteurs d'OSMIA à diffuser ce document au plus grand nombre. Les articles originaux proposés à la publication sont systématiquement soumis à deux relecteurs avant leur publication pour assurer cohérence et rigueur aux informations publiées.

Nous espérons que vous prendrez autant de plaisir à parcourir OSMIA que nous en avons eu à le préparer et nous vous invitons à nous faire part de vos suggestions (nicovereecken@yahoo.fr) tant au sujet du fond que de la forme, de manière à nous permettre de perfectionner OSMIA au fil du temps.

Bonnes observations à toutes et à tous et à très bientôt pour un prochain numéro!



